\title{
CHIRAL PROPERTY OF FIELD GALAXIES AROUND THE LOCAL SUPERCLUSTER
}

\author{
Binil Aryal* \\ *Central Department of Physics, Tribhuvan University, Kirtipur, Nepal.
}

\begin{abstract}
We present an analysis of the winding sense of 1,621 field galaxies having radial velocity $3000 \mathrm{~km} / \mathrm{s}$ to 5,000 km/s. The arm patterns ( $\mathrm{S}$ and Z-shaped, i.e., chiral property) of the spirals are visually studied. In order to classify the structural modes of spirals, we assume a random direction of the rotation of galaxies. Anisotropy in the chiral symmetry and the preferred alignment of galaxies is studied using chi-square, auto-correlation and the Fourier tests. We noticed a good agreement between the random alignment of the position angle (PA) distribution and the existence of chirality in both the leading and trailing modes. Chirality is found stronger for the late- type spirals (Sc, Scd, Sd and Sm) than that of the early types (Sa, Sab, Sb and Sbc). A significant dominance (17\% \& 8.5\%) of trailing modes is noticed in the barred spirals. In addition, chirality of field galaxies is found to remain invariant under the global expansion. A random alignment is noticed in the PA-distribution of leading and trailing mode spirals.
\end{abstract}

Key Words: Spiral galaxies; Hubble sequence; Chirality; Clusters: individual (Local Supercluster).

\section{INTRODUCTION}

An object that is not superimposable on its mirror image is termed as chiral object, such as the letter "E" and its mirror image. Chiral objects do not show reflection symmetry, but may exhibit rotational symmetry. They are not necessarily asymmetric, because they can have other types of symmetry. An object is achiral (non chiral) if and only if it has an axis of improper rotation (rotation by $360^{\circ} / \mathrm{n}$ ) followed by a reflection in the plane perpendicular to this axis. Thus, an object is chiral if and only if it lacks such an axis.

Chiral symmetry breaking or restoration is a key ingredient in different problems of theoretical physics: from nonperturbative quantum cromodynamics to highly doped semiconductors $^{1}$. For a pseudoscalar meson, it is found that the chiral symmetry breaking decreases with increasing current quark mass ${ }^{2}$. Bagchi et al. (2006) studied the large color approximation of the compact strange stars and discussed the chiral symmetry restoration ${ }^{3}$. They claimed that the chiral symmetry restoration can be understood by exploring the possible existence of strange stars in the Universe. In principle, microscopic process triggered huge astrophysical large scale structure ${ }^{4}$. It is therefore interesting to study the breaking or restoration of chiral symmetry not only in microscopic phenomena (L-neutrino, mesons, Laminoacids, D-sugars, etc), but also in macroscopic ones (stars, molecular clouds, galaxies). According to the basic concepts of cosmology, initial quantum fluctuations have been hugely enhanced during the inflationary epoch, leading the formation of the large scale structure in the Universe ${ }^{5}$. Thus, the macroscopic chirality should be related to some primordial microscopic process which led to the today's observed large scale structures ${ }^{6}$.

Pasha (1985) used 'tilt' criteria and studied the sense of winding of the arms in 132 spirals and confirmed 107 spirals to have trailing $\mathrm{arm}^{7}$. It should be remembered that the classical 'tilt' criteria is based on the visible asymmetry of a dust matter distribution. Now it is believed that the dark matter halo dominates the dynamics of the galaxies throughout. Ostriker et al. (1974) predicted that the dark matter is concentrated in the extended galactic halos ${ }^{8}$. The nature of dark matter in the galactic halo of spiral galaxies is still undetermined. The significant role of DM haloes can not be denied in the context of hierarchical galaxy formation. In this context, we are afraid that the visible study of the dust lanes might mislead the reality. Thomasson et al. (1989) studied theoretically and performed N-body simulations in order to understand the formation of spiral structures in retrograde galaxy encounters. They concluded that the formation process for the leading and trailing pattern is completely different ${ }^{9}$. Interestingly, they noticed the importance of halo mass in both the cases. Trailing spiral arms are generated by swing amplification ${ }^{10}$. Since the swing amplifier is very powerful, only small perturbations are necessary for a strong two armed trailing pattern to develop. They found that the amount of amplification depends on the halo-to-disk mass ratio. The formation mechanism of leading arm spiral does not depend on self gravity. The leading arms are expected to dominate over the trailing ones in galaxies with large $\backslash$ haloes, where the swing amplifier is almost dead. Thomasson et al. (1989) concluded that the spirals having halos with masses larger than the disk mass exhibit leading pattern. The existence of companion and the merging process 
can be seen everywhere in the large scale structure (clusters as well as in the field). The role of dark matter halo might reveal the actual dynamics of the structure formation in the future. Sugai \& Iye (1995) used statistics and studied the winding sense of galaxies (S- and Z-shaped) in 9,825 spirals ${ }^{11}$. No significant dominance from a random distribution is noticed. Aryal \& Saurer (2005a) studied the spatial orientations of spin vectors of 4,073 galaxies in the Local Supercluster ${ }^{12}$. Again, no preferred alignment is found. These results hint that the distribution of angular momentum of galaxies is found to be random in two- (S- and Z-shaped) and three-dimensional (spin vector) analysis provided the database is rich and complete. Thus, one can assume that the choice of rotation for a galaxy might be random for an observer.

By considering the group of transformations acting on the configuration space, Capozziello \& Lattanzi (2006) revealed that the spiral galaxies possess chiral symmetry in the large scale structure ${ }^{13}$. In addition, they predicted that the progressive loss of chirality might have some connection with the rotationally-supported (spirals, barred spirals) and randomized stellar systems (lenticulars, ellipticals). Aryal, Acharya \& Saurer (2007) carried out a study to test the Capozziello and Lattanzi's (2006) prediction regarding the progressive loss of chirality in the large scale structure ${ }^{14}$. They analysed the distribution of leading and trailing arm galaxies in the Local Supercluster (LSC) and concluded the existence of chiral symmetry for both the spirals and the barred spirals. However, the Virgo cluster galaxies show a preferred alignment: the galactic rotation axes of leading and trailing structures are found to lie in the equatorial plane. Aryal \& Saurer (2005a) noticed a preferred alignment for the late-type spirals and barred spirals in the Local Supercluster. In addition, they found that the spin vector projections of earlyand late-type galaxies show opposite alignment. Their results hint the existence of the chiral characterization.

In this work, we present an analysis of chiral property of field galaxies having radial velocity (RV) 3,000 km/s to 5,000 km/s. We intend to study the importance of chiral symmetry in order to understand the structural modes of the galaxy. In addition, we expect to study the following: (1) Does chirality exist for the spirals and barred spirals in the field? (2) Is there any correlation between the chirality and preferred alignment of galaxies? (3) Does the group of galaxies exhibit chiral characterization? and finally, (4) What can we say about the chiral and achiral (non-chiral) properties of the large scale structure?

This paper is organized as follows: in Sect. 2 we describe the method of data reduction. In Sect. 3 we give a brief account of the methods and the statistics used. Finally, a discussion of the statistical results and the conclusions are presented in Sects. 4 and 5.

\section{THE SAMPLE: DATA REDUCTION}

The method of data compilation and the identification of the structural modes of the galaxies were the same as in Aryal, Acharya \& Saurer (2007) ${ }^{14}$. We describe the selection criteria and the data reduction process in brief here. A galaxy had to fulfill the following selection criteria in order to be selected: (1) the RV should lie in the range $3,000 \mathrm{~km} / \mathrm{s}$ to $5,000 \mathrm{~km} / \mathrm{s}$, (2) the morphology should be known, (3) should not be the cluster galaxy, (4) the diameters, magnitude and the position angle should be given and, (5) the patterns of the arms ( $\mathrm{S}$ and Z-shaped) should be visible.

There were two clusters Abell 0426 $\left(\mathrm{RA}(\mathrm{J} 2000)=03^{\circ} 18^{\mathrm{m}} 36.4^{\mathrm{s}}\right.$, $\left.\operatorname{Dec}(J 2000)=+41^{\circ} 30^{\prime} 54^{\prime \prime}\right)$ and Abell 3627 (RA(J2000) = $16^{\mathrm{h}} 15^{\mathrm{m}} 32.8^{\mathrm{s}}$, Dec. (J2000) $\left.=-60^{\circ} 54^{\prime} 30^{\prime \prime}\right)$ in our region. These clusters have mean RVs 5,366 km/s (75 $\pm 5 \mathrm{Mpc}$ ) and 4,881 $\mathrm{km} / \mathrm{s}(63 \pm 4 \mathrm{Mpc})$, respectively ${ }^{15,16}$. We removed the galaxies belong to the cluster Abell 0426 using the catalog established by Brunzendorf \& Meusinger (1999) ${ }^{17}$. For the cluster Abell 3627 galaxies, we used Photometric Atlas of Northern Bright Galaxies (Kodaira, Okamura, \& Ichikawa 1990) and Uppsala Galaxy Catalogue (Nilson 1973) ${ }^{18,19}$. There were 174 galaxies belongs to these clusters in our database.
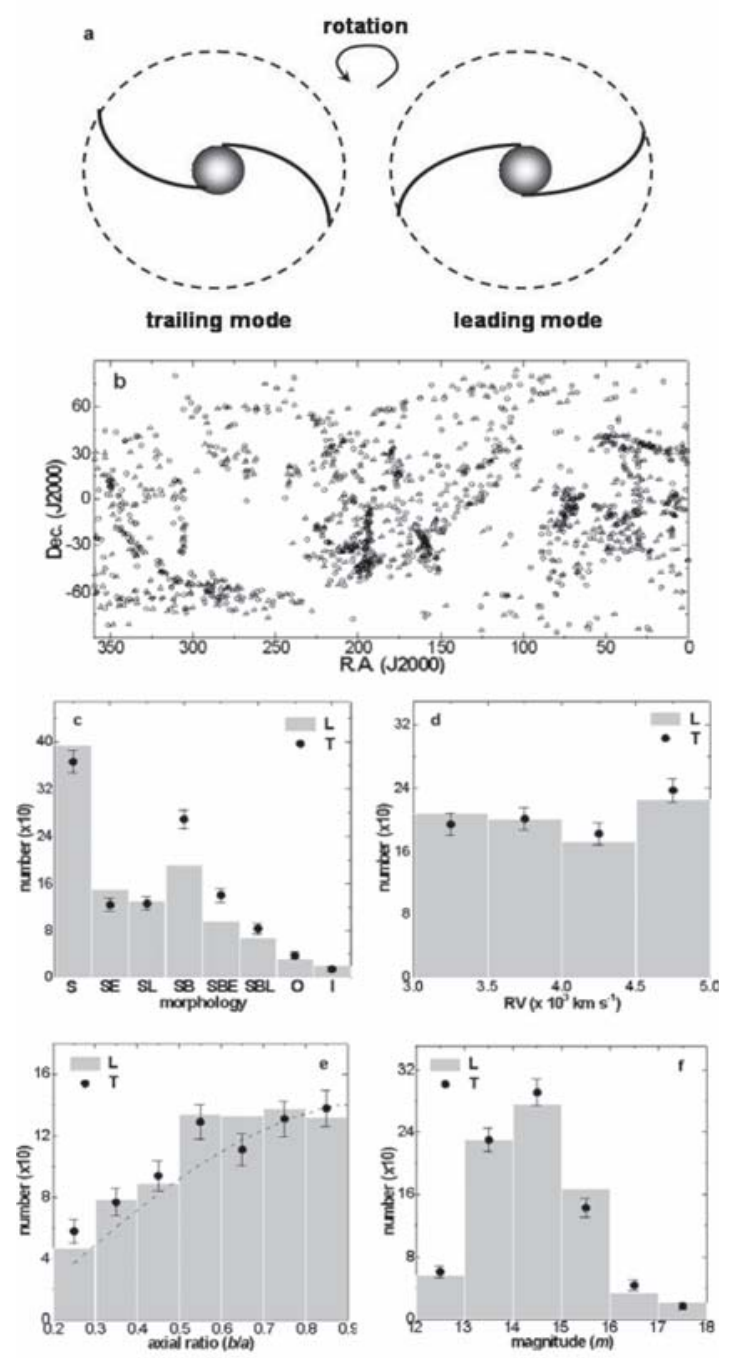

Figure 1: (a) A sketch representing the orientation (leading and trailing modes) of the spiral arms relative to the direction of rotation of the galaxy. (b) All-sky distribution of 1,621 leading (triangle) and trailing (circle) arm field galaxies having RVs in the range $3,000 \mathrm{~km} / \mathrm{s}$ to $5,000 \mathrm{~km} / \mathrm{s}$. The morphology (c), radial velocity (d) distribution of leading and trailing arm galaxies in our databse. The statistical $\pm 1 \sigma$ error bars are shown for the trailing subsample. 
The RVs were compiled from Las Campanas Redshift Survey (Shectman 1996) ${ }^{20}$. The PAs and the diameters of galaxies were added from the Uppsala Galaxy Catalogue (Nilson 1973), Uppsala obs. General Catalogue, Addendum (Nilson 1974), Photometric Atlas of Northern Bright Galaxies (Kodaira, Okamura, I\& Ichikawa 1990), ESO/Uppsala Survey of the European Southern Observatory (Lauberts 1982), Southern Galaxy Catalogue (Corwin et al. 1985) and Third Reference Catalogue of Bright Galaxies (de Vaucouleurs et al. $1991)^{18,19,21,22,23,24}$.

In the NED (NASA/IPAC extragalactic database, http:// nedwww.ipac.caltech.edu/), 6,493 galaxies having RVs 3,000 $\mathrm{km} / \mathrm{s}$ to $5,000 \mathrm{~km} / \mathrm{s}$ were listed until the cutoff date (October 2009). The diameters were given for 5,324 (82\%) galaxies. Both the diameters and PAs were listed for 3,971 (61\%) galaxies. Morphological information was given in the catalogues for $3 \backslash, 276$ (50\%) galaxies. We inspected the DSS images of 3,276 galaxies using NED and ALADIN2.5 softwares. There were 2,107 (32\%) galaxies having distinct spiral arms in our database.

It is true that the determination of the direction of rotation of the galaxies is a difficult problem. One can distinguish the arm patterns (S- and Z-shaped) visually. Without knowing actual rotation, it is impossible to identify true structural mode. We argue here that the choice of rotation for a galaxy should be random. We used computer and gave random direction to our galaxies. Thus, each galaxy got a virtual direction by the computer. In this way, we identified structural modes of galaxies in our database. For individual galaxies, these structural modes might be incorrect. Statistically, the results might be important in the subjective sense. It should be noticed that the authors of this paper are investigating spatial alignments of galaxies in a variety of cosmic environments (Aryal \& Saurer 2004, 2005a, 2005b, 2006, 2007) 25,26,27,28. In general the results of these works support the hierarchical clustering scenario (Peebles, 1969) which predicts that the directions of the spin vectors are entirely random ${ }^{29}$. While analysing spatial orientation of spin vectors of galaxies, we have assumed a spatial isotropic distribution as a theoretical reference. Our observations are compared with expected isotropic distribution curves.

The Digitized Sky Survey (DSS) image and the contour maps of the galaxies were studied in order to identify ' $S$ ' and ' $Z$ ' shape of the galaxies. For this, we used MIDAS software. Fig. 1a shows the leading and trailing modes if the rotation is counterclockwise. The re-examination of the arms using IRAF software resulted the rejection of more than $23 \%$ of the objects. These rejected galaxies were nearly edge-on galaxies. As expected, it was relatively easier to identify the winding patterns (S \& Z shape) of nearly face-on than that of nearly edge-on galaxies. These patterns are used to classify leading and trailing modes assuming random direction for the rotation.\} In this way, we compiled a database of 1,621 galaxies showing either leading or trailing structural mode. There were 807 leading and 821 trailing patterns in our database. All sky distribution of these patterns is shown in Fig. 1b. The symbols "circle" and "triangle" represent the positions of the trailing and the leading patterns, respectively. Several groups and aggregations of the galaxies can be seen in the figure. The inhomogeneous distribution of the positions of the galaxies might be the selection effects for the galaxy orientation study (Aryal \& Saurer 2000) ${ }^{31}$.

The morphology, radial velocity, distributions of leading and the trailing arm field galaxies are shown in Figs. 1. The spirals (47\%) dominate our database in the morphological distribution (Fig. 1c). However, a significant dominance of trailing modes are noticed in the barred spirals whereas a weak dominance of leading modes are found in the spirals. The galaxies in the $R V$ distribution $(\Delta R V=5,00 \mathrm{~km} / \mathrm{s}$ ) were nearly equal (Fig. 1d).

We classified the database into 34 subsamples for both the leading and trailing modes on the basis of the morphology, radial velocity, area and the group of the galaxies. A statistical study of these subsamples are given in Table 1 and discussed in Sect. 3.1.

\section{METHOD OF ANALYSIS}

Basic statistics is used to study the dominance of leading and trailing modes. At first, morphology and RV dependence is studied concerning the chiral property of galaxies. Secondly, sky is divided into 16 equal parts in order to observe the violation of chirality locally. Several galaxy groups are identified in the all-sky map. Finally, we study the anisotropic distribution of the leading or trailing modes in these groups. For a subsample to show chiral symmetry, we set the difference between the number of leading and trailing modes, $\Delta<5 \%$. The chiral symmetry is assumed to be violated when $\Delta>5 \%$.

We assume isotropic distribution as a theoretical reference and studied the equatorial PA-distribution in the total sample and subsamples. In order to measure the deviation from isotropic distribution we have carried out three statistical tests: chi-square, auto-correlation and the Fourier.

We set the chi-square probability $\mathrm{P}\left(>\chi^{2}\right)=0.050$ as the critical value to discriminate isotropy from anisotropy, this corresponds to a deviation from isotropy at the $2 \sigma$ level (Godlowski 1993) ${ }^{32}$. Auto correlation test takes account the correlation between the numbers of galaxies in adjoining angular bins. We expect, auto correlation coefficient C'!0 for an isotropic distribution. The critical limit is the standard deviation of the correlation coefficient $\mathrm{C}$.

If the deviation from isotropy is only slowly varying with angles (in our case: PA) the Fourier test can be applied.

Here the Fourier coefficients $\Delta_{11}$ and $\Delta_{21}$ are the parameters of the distributions. We obtain the following expressions for the Fourier coefficients $\Delta_{11}$ and $\Delta_{21}$

$$
\begin{gathered}
\Delta_{11}=\frac{\sum_{k=1}^{n}\left(N_{k}-N_{0 k}\right) \cos 2 \theta_{k}}{\sum_{k=1}^{n} N_{0 k} \cos ^{2} 2 \theta_{k}} \\
\Delta_{21}=\frac{\sum_{k=1}^{n}\left(N_{k}-N_{0 k}\right) \sin 2 \theta_{k}}{\sum_{k=1}^{n} N_{0 k} \sin ^{2} 2 \theta_{k}}
\end{gathered}
$$


The standard deviations $\sigma\left(\Delta_{11}\right)$ and $\sigma\left(\Delta_{\square 1}\right)$ can be obtained using the expressions

$$
\begin{aligned}
& \sigma\left(\Delta_{11}\right)=\left(\sum_{k=1}^{n} N_{0 k} \cos ^{2} 2 \theta_{k}\right)^{-1 / 2} \\
& \sigma\left(\Delta_{21}\right)=\left(\sum_{k=1}^{n} N_{0 k} \sin ^{2} 2 \theta_{k}\right)^{-1 / 2}
\end{aligned}
$$

The probability that the amplitude

$$
\Delta_{1}=\left(\Delta_{11}{ }^{2}+\Delta_{21}{ }^{2}\right)^{1 / 2}
$$

is greater than a certain chosen value is given by the formula

$$
P\left(>\Delta_{1}\right)=\exp \left(-\frac{n}{4} N_{0} \Delta_{1}^{2}\right),
$$

with standard deviation $\sigma\left(\Delta_{1}\right)=\left(\frac{2}{n N_{0}}\right)^{1 / 2}$

The Fourier coefficient $\Delta_{11}$ is very important because it gives the direction of departure from isotropy. In the analysis of the position angle (PA), if $\Delta_{11}<0$ an excess of galaxies with rotation axis parallel to the reference plane is present, whereas for $\Delta_{11}>0$ the rotation axis tend to be perpendicular to that plane. The first order Fourier probability function $P\left(>\Delta_{1}\right)$ estimates the depth of the preferred orientation. The first order Fourier probablity function $P\left(>\Delta_{11}\right)$ estimates whether (smaller value of $P\left(>\Delta_{1}\right)$ ) or not (higher value of $P\left(>\Delta_{1}\right)$ ) a pronounced preferred orientation occurs in the sample. We set $P\left(>\Delta_{11}\right)=0.150$ as a critical value.

\section{RESULT}

First we present the statistical result concerning the anisotropic distribution of leading and trailing patterns of galaxies in the total sample and subsamples. Then, we repeat the same in the limited region of the sky and in the groups. The equatorial PA-distribution of galaxies in the total sample and subsamples is presented. At the end, a general discussion and a comparison with the previous results will be presented.

\section{Distribution of leading and trailing structures}

A statistical comparison between the total sample and subsamples of the leading ( $\mathrm{L}$, hereafter) and trailing $(\mathrm{T}$, hereafter) modes is given in Table 1. Fig. 2 shows this comparison in the histogram. The $\Delta(\%)$ in Table 1 and Fig. 2 represent the percentage difference between $\mathrm{L} \& \mathrm{~T}$ modes. We studied the standard deviation (sde) of the major diameters (a) of galaxies in the total sample and subsamples for both $\mathrm{L}$ \& T modes. In Table $1, \Delta$ (a sde) represents the difference between the standard deviation of the major diameters of $L$ \& T modes.

An insignificant difference $(0.4 \% \pm 0.2 \%)$ between $\mathrm{L} \& \mathrm{~T}$ modes $\}$ are found in the total sample (Table 1$)$. The difference between the standard deviation of the major diameters ( $\Delta$ (a sde)) of L \& T modes is found less than 0.019 (seventh column,
Table 1). Interestingly, the sum of the major diameters of total L \& T mode galaxies coincides. This result suggests the existence of chirality in the field galaxies having RV 3,000 km/ $\mathrm{s}$ to $4,000 \mathrm{~km} / \mathrm{s}$.

In Fig. 2, the slanting-line (grey-shaded) region corresponds to the region showing $\leq 10 \%(5 \%) \Delta$ value. Almost all subsamples lie within this region, suggesting the existence of chirality within $10 \%$ error limit. We present the distribution in the subsamples of $\mathrm{L} \& \mathrm{~T}$ mode galaxies classified according as their morphology, RVs, area and the groups below.

\section{Morphology}

In the spirals, $\mathrm{L}$ modes are found $3.7 \%( \pm 1.8 \%)$ more than that of T modes. The chirality is found stronger for the latetype spirals (Sc, Scd, Sd and Sm) than that of early-type (Sa, Sab, Sb and Sbc): $\Delta$ value turned out to be $9.5 \%( \pm 4.8 \%)$ and $1.8 \%( \pm 1.0 \%)$ for early- and late-types (Table 1$)$. Thus, the late-type spirals are the best candidate of the chiral object in our database.
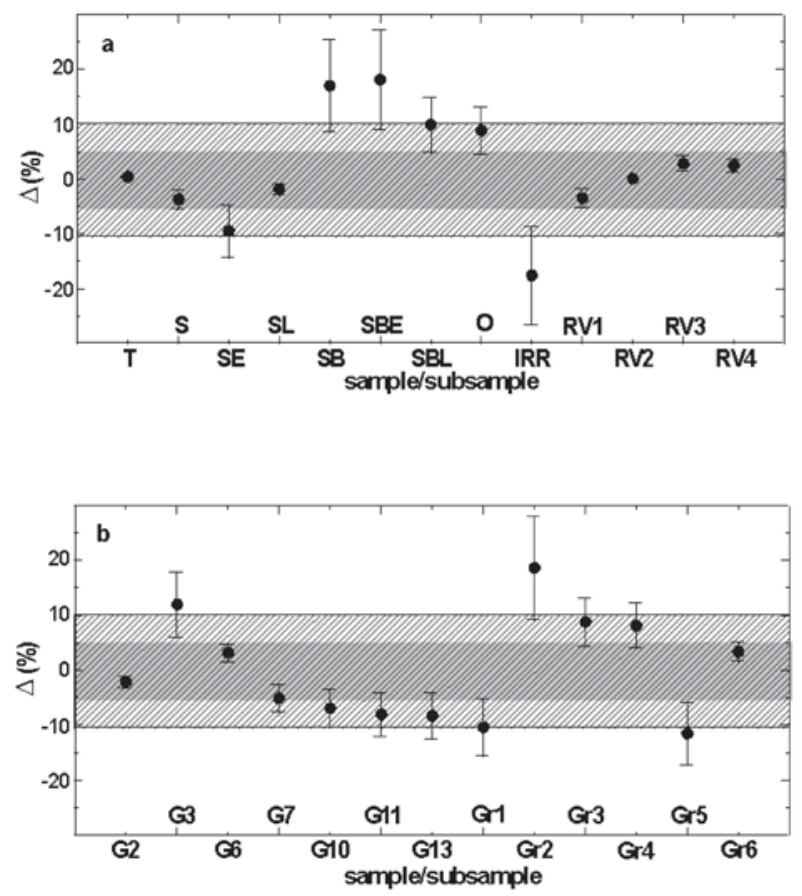

Figure 2: Statistics of the leading and trailing modes of the galaxies in the total and subsamples. The full form of the abbreviations (Xaxis) is given in Table 1 (first column). $\Delta(\%)=\mathrm{T}-\mathrm{L}$, where $\mathrm{T}$ and $\mathrm{L}$ represent the number of trailing and leading arm galaxies, respectively. The statistical error bars $\sigma(\%)$ shown in the figure are calculated as: $\sigma(\%)=\sigma /\left(T^{0.5}+L^{0.5}\right) \times 100$, where $\sigma=\left(T^{0.5}-L^{0.5}\right)$. The grey-shaded and the slanting-line region represent the $< \pm 5 \%$ and $< \pm 10 \% \Delta$ value, respectively.

The dominance of $\mathrm{T}$ modes are found to be significant $(17 \% \pm$ $8.5 \%$ ) in the spirals. The $\Delta$ value is found $>9 \%$ for both early(SBa, SBab, SBb and SBbc) and late-type (SBc, SBcd, SBd, $\mathrm{SBm}$ ) barred spirals. Thus, we suspect that the field SB galaxies are not the best candidates of chiral objects. Similar result (i.e., $\Delta>8 \%$ ) is found for the irregulars and the morphologically unidentified galaxies. One interesting similarity is noticed 
Table 1: Statistics of leading (column 3) and trailing modes (column 4 ) in the total sample and subsamples. The fifth and sixth column give the numeral and percentage difference $(\Delta=\mathrm{T}-\mathrm{L})$ between the trailing $(\mathrm{T})$ and the leading $(\mathrm{L})$ structural modes. The next two columns give the error: $\sigma=\left(\mathrm{T}^{0.5}-\mathrm{L}^{0.5}\right)$ and $\sigma(\%)=\sigma /\left(\mathrm{T}^{0.5}+\mathrm{L}^{0.5}\right) \times 100$. The eighth column gives the difference between the standard deviation (in arc minute) of the major diameters $(a)$ of the trailing and leading modes in the galaxies $(\Delta(a$ sde $))$. The difference between the sum of the major diameters $(\Delta(a) \%)$ are listed in the last column. The sample/ subsample and their abbreviations are given in first two columns.

\begin{tabular}{|c|c|c|c|c|c|c|c|c|}
\hline sample/subsample & symbol & $\mathrm{L}$ & $\mathrm{T}$ & $\Delta$ & $\Delta(\%)$ & $\sigma(\%)$ & $\Delta$ (a sde $)$ & $\Delta(a)(\%)$ \\
\hline Total & & 814 & 807 & 7 & 0.4 & 0.2 & 0.019 & 0.0 \\
\hline Spiral & & 395 & 367 & -28 & -3.7 & $7-1.8$ & 0.031 & 3.0 \\
\hline Spiral (early-type) & SE & 150 & 124 & -26 & -9.5 & $5-4.8$ & 0.058 & 8.1 \\
\hline Spiral (late-type) & SL & 131 & 126 & -5 & -1.9 & $9-1.0$ & 0.031 & 0.3 \\
\hline Barred Spiral & $\mathrm{SB}$ & 191 & 269 & 78 & 17.0 & $0 \quad 8.5$ & 0.062 & 15.2 \\
\hline Barred Spiral (early-type) & SBE & 97 & 140 & 43 & 18.1 & 19.1 & .091 & 14.6 \\
\hline Barred Spiral (late-type) & SBL & 68 & 83 & 15 & 9.9 & 95.0 & 0.066 & 9.2 \\
\hline Unknown Morphology & 0 & 31 & 37 & 6 & 8.8 & $8 \quad 4.4$ & .095 & 3.9 \\
\hline Irregular & & 20 & 14 & -6 & -17.6 & $6-8.9$ & 0.105 & 5.2 \\
\hline $3000<\mathrm{RV}\left(\mathrm{km} \mathrm{s}^{-1}\right) \leq 3500$ & RV1 & 194 & 208 & -14 & -3.5 & $5-1.7$ & 0.046 & 2.6 \\
\hline $3500<\mathrm{RV}\left(\mathrm{km} \mathrm{s}^{-1}\right) \leq 4000$ & RV2: & 201 & 201 & 0 & 0.0 & 00.0 & 0.031 & 3.1 \\
\hline $4000<\mathrm{RV}\left(\mathrm{km} \mathrm{s}^{-1}\right) \leq 4500$ & RV3 & 182 & 172 & 10 & 2.8 & $8 \quad 1.4$ & 0.034 & 0.6 \\
\hline $4500<\mathrm{RV}\left(\mathrm{km} \mathrm{s}^{-1}\right) \leq 5000$ & RV4: & 237 & 226 & 11 & 2.4 & 1.2 & 0.041 & 1.0 \\
\hline Grid 1 & G1 & 21 & 20 & 1 & 2.4 & $\begin{array}{ll}4 & 1.2\end{array}$ & 68 & 6.8 \\
\hline & G2 & 116 & 121 & -5 & -2.1 & $1-1.1$ & 14 & 1.6 \\
\hline Gric & G3 & 112 & 88 & 24 & 12.0 & $\begin{array}{ll}0 & 6.0\end{array}$ & 76 & 9.3 \\
\hline Grid & G4 & 14 & 12 & 2 & 7.7 & $\begin{array}{ll}7 & 3.9\end{array}$ & 17 & 9.7 \\
\hline & G5 & 8 & 11 & -3 & -15.8 & $8-7.9$ & & 22.3 \\
\hline Gri & G6 & 80 & 75 & 5 & 3.2 & $2 \quad 1.6$ & 81 & 4.5 \\
\hline & G7 & 56 & 62 & -6 & -5.1 & $1-2.5$ & & 8.9 \\
\hline & G8 & 33 & 22 & 11 & 20.0 & $\begin{array}{lll}0 & 10.1\end{array}$ & 73 & 12.9 \\
\hline & G9 & 31 & 20 & 11 & 21.6 & $\begin{array}{ll}6 & 10.9\end{array}$ & & 18.1 \\
\hline & G10 & 108 & 124 & -16 & -6.9 & $9-3.5$ & 04 & 5.4 \\
\hline & G11 & 52 & 61 & -9 & -8.0 & $0-4.0$ & 25 & 6.2 \\
\hline & G12 & 20 & 20 & 0 & 0.0 & $\begin{array}{ll}0 & 0.0\end{array}$ & & 7. \\
\hline & G13 & 66 & 78 & -12 & -8.3 & $3-4.2$ & 39 & 2.9 \\
\hline & G14 & 44 & 37 & 7 & 8.6 & $\begin{array}{ll}6 & 4.3\end{array}$ & & 10.3 \\
\hline & G15 & 44 & 47 & -3 & -3.3 & $3-1.6$ & 50 & 5. \\
\hline & G16 & 9 & 9 & 0 & 0.0 & $\begin{array}{ll}0 & 0.0\end{array}$ & & 8.6 \\
\hline & Gr1 & 30 & 37 & -7 & -10.4 & $4-5.2$ & & 7.1 \\
\hline & Gr2 & 70 & 48 & 22 & 18.6 & $\begin{array}{ll}6 & 9.4\end{array}$ & 0.097 & 12.6 \\
\hline & Gr3 & 37 & 31 & 6 & 8.8 & $\begin{array}{ll}8 & 4.4\end{array}$ & & 4.3 \\
\hline & $\mathrm{Gr} 4$ & 40 & 34 & 6 & 8.1 & $\begin{array}{ll}1 & 4.1\end{array}$ & & 3.9 \\
\hline & Gr5 & 85 & 107 & -22 & -11.5 & $5-5.7$ & 0.089 & 11.2 \\
\hline Group 6 & Gr6 & 45 & 42 & 3 & 3.4 & $\begin{array}{ll}4 & 1.7\end{array}$ & 0.024 & 1.6 \\
\hline
\end{tabular}

between the late-type spirals and barred spirals. The $\Delta$ value for both the late-types are found less than that of early-types (see Table 1). Thus, the chirality is favourable for the latetypes rather than the early-types. The difference between the standard deviation of the major diameters $\Delta(a$ sde) for $\mathrm{L} \& \mathrm{~T}$ modes is found less than 0.050 arc minute for the total sample, spirals and the late-type spirals (seventh column, Table 1). These samples showed $\Delta$ value $<5 \%$ (grey-shaded region, Fig. 2a). Thus, we found a good correlation between the $\Delta(\%)$ and $\Delta(a$ sde $)$ value. Probably, this result hints the fact that the size of the non-superimposable mirror images should lie within a limit. In our database, this limit should not exceed 0.050 arc minute for $\Delta($ a sde).

The difference between the sum of the major diameters (in percentage) are found greater than $10 \%$ for the barred spirals and early-type barred spirals. Interestingly, these two subsamples showed $\Delta$ value greater than $15 \%$ (Fig. 2a). We suspect that the SB galaxies possess chiral symmetry breaking.

\section{Radial velocity}

A very good correlation between the numbers of $\mathrm{L} \& \mathrm{~T}$ modes can be seen in the RV classifications (Fig. 1d). All 4 subsamples show the $\Delta$ and $\Delta(a$ sde) value less than $5 \%$ and 0.050 , respectively (Table 1 ). In addition, $\Delta(a)$ is found to be $<5 \%$. This result is important in the sense that the statistics in these subsamples is rich (number of galaxies $>170$ ) enough. However, a difference is noticed: dominance of $\mathrm{L} \& \mathrm{~T}$ modes in low (RV1) and high (RV3, RV4) RV subsamples, respectively. However, this dominance has no meaning because $\Delta<5 \%$. An equal number of $L \&$ T modes are found in the subsample $\operatorname{RV} 2(3,500<\mathrm{RV}(\mathrm{km} / \mathrm{s}) \leq 4,000)$ (Table 1$)$. In order to check the binning effect, we further classify the total galaxies in $6(\Delta \mathrm{RV}$ $=333 \mathrm{~km} / \mathrm{s})$ and 8 bins $(\Delta \mathrm{RV}=250 \mathrm{~km} / \mathrm{s})$ and study the statistics. No significant dominance of either $\mathrm{L} \& \mathrm{~T}$ modes is noticed. Thus, we noticed that the chiral property of field galaxies remain invariant with the global expansion (i.e, expansion of the Universe). This result might be important.

\section{Area}

We study the distribution of L \& T modes by dividing the sky into 16 equal parts (Fig. 3a). The area of the grid (G) is $90^{\circ}$ $x 45^{\circ}$ (RA x Dec). The area distribution of $L \&$ T modes are plotted, that can be seen in Fig. 3a'. The statistical parameters are given in Table 1 . A significant dominance $(>2 \sigma)$ of T mode is noticed in grid 3 (RA: $0^{\circ}$ to $90^{\circ}$, Dec: $0^{\circ}$ to $45^{\circ}(\mathrm{J} 2000)$ ) (Fig. 3a,a'). An elongated subcluster like structure can be seen in this grid. In this grid, $\Delta, \Delta(a$ sde $)$ and $\Delta(a)$ are found to be $12 \% \pm 6 \%, 0.076$ and $9.3 \%$, respectively. These figures suggest that the galaxies in G3 lost their chiral property.
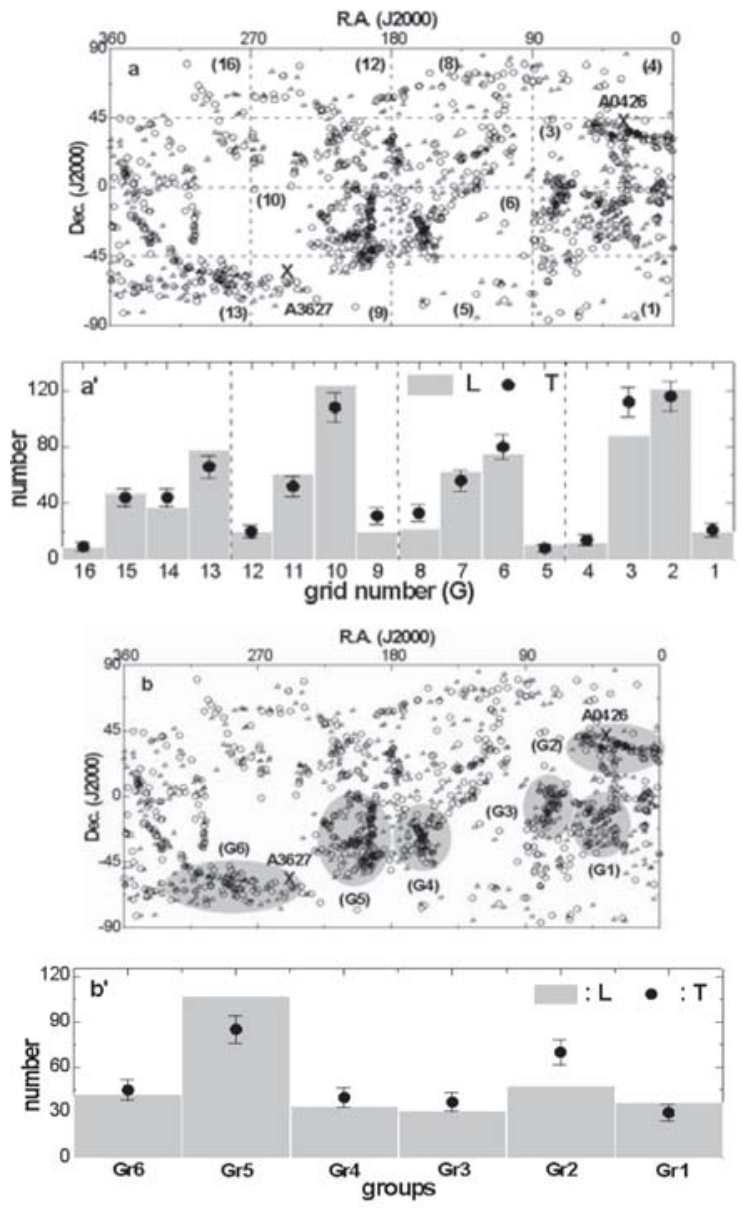

Figure 3: (a) All sky distribution of leading (hollow circle) and trailing (hollow triangle) modes in 16 area grids. (a') The histogram shows the distribution of the L \& T modes in the grids G1 to G16. (b) Six groups of the galaxies, represented by the grey-shaded region. (b') The distribution of $\mathrm{L} \& \mathrm{~T}$ modes in 6 groups. The statistical error bar $\pm 1 \sigma$ is shown. The positions of the clusters Abell 0426 and Abell 3627 are shown by the symbol " $\mathrm{x}$ ” (a,b). 
A dominance $(\sim 1.5 \sigma)$ of $\mathrm{L}$ mode is noticed in G10 (RA: $180^{\circ}$ to 270 ${ }^{\circ}$, Dec: $-45^{\circ}$ to $0^{\circ}$ (J2000)) and G13 (RA: $270^{\circ}$ to $360^{\circ}$, Dec: $90^{\circ}$ to $-45^{\circ}(\mathrm{J} 2000)$ ) (Fig. 3a,a'). In both the grids, a large aggregation of the galaxies can be seen. A subcluster-like aggregation can be seen in G10. An elongated structure can be seen in G13. In both the grids, $\Delta$ value is found to be greater than $5 \%$ (Table 1$)$. Thus, we suspect that the galaxies in these grids (G10, G13) are loosing chiral symmetry. This result might reveal the effects of the cluster evolution on chiral symmetry of galaxies. No dominance of either $L$ or $T$ modes is noticed in the groups G1, G2, G4, G5, G6, G7, G11, G12, G14, G15 and G16. Thus, the chirality is found intact in $80 \%$ area of the sky. We suspect that the groupings or subclusterings of the galaxies lead the violation of chirality in the grids G3, G10 and G13. We study the existence of chirality in the groups below.

\section{Galaxy groups}

In all-sky map, several groups of galaxies can be seen (Fig. 3a). It is interesting to study the existence of chirality in these groups. For this, we systematically searched for the groups fulfilling following selection criteria: (a) major diameter $>30^{\circ}$, (b) cutoff diameter $<2$ times the background galaxies, (c) number of galaxies $>50$. We found 6 groups fulfilling these criteria (Fig. 3b). All 6 groups (Gr) are inspected carefully. In 3 groups (Gr2, Gr5 and Gr6), subgroups can be seen. The number of galaxies in the groups Gr2 and Gr5 are found more than 100 .

The clusters Abell 0426 and Abell 3627 are located close to the groups Gr2 and Gr6. The symbol " $x$ ” represents the cluster center in Fig. 3b. The mean radial velocities of these clusters are $5,366 \mathrm{~km} / \mathrm{s}$ and 4,881 km/s, respectively. However, we have removed the member galaxies of these clusters from our database. A significant dominance $(>2 \sigma)$ of T mode is noticed in the group Gr2 (Fig. 3b,b'). The $\Delta, \Delta(a$ sde) and $\Delta(a) \%$ values are found to be $18.6 \% \pm 9.4 \%, 0.097$ and $12.6 \%$, respectively (Table 1). These values indicate that the galaxies in this group might lose their chiral symmetry. We suspect that the galaxies in this group are under the influence of the cluster Abell 0426, due to which apparent subclustering of the galaxies can be seen in this group. This subclustering leads the violation of chiral symmetry.

The galaxies in Gr5 shows an opposite preference: a significant dominance of the $\mathrm{L}$ mode $(>2 \sigma)$ (Fig. 3b,b'). In this group, $\Delta, \Delta(a$ sde $)$ and $\Delta(a) \%$ are found to be $11.5 \% \pm 5.7 \%$, 0.089 and $11.2 \%$, suggesting the violation of chirality (Table 1). No humps or dips can be seen in the groups Gr1, Gr3, Gr4 and Gr6 (Fig. 3.2b,b'). Thus, we conclude that the large aggregation of the galaxies lead the violation of chirality.

\section{Anisotropy in the position angle distribution}

We study the equatorial position angle (PA) distribution of L \& T modes in the total sample and the subsamples. A spatially isotropic distribution is assumed in order to examine nonrandom effects in the PA-distribution. In order to discriminate the deviation from the randomness, we use three statistical tests: chi-square, auto correlation and the Fourier. The bin
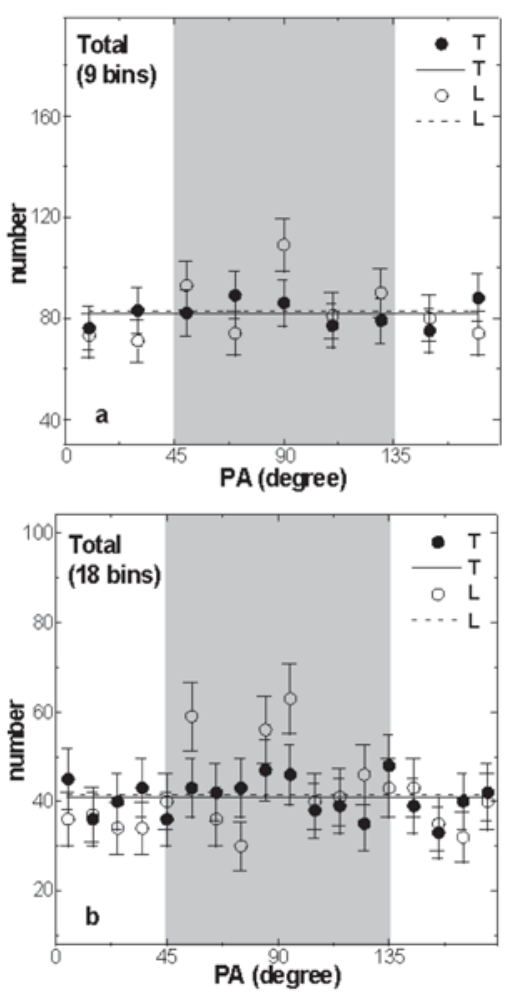

Figure 4: The equatorial position angle (PA) distribution of L \& T modes in the total sample. The histograms are plotted in 9 (a) and 18 (b) bins. The solid and the dashed line represent the expected isotropic distribution for $\mathrm{L} \& \mathrm{~T}$, respectively. The observed counts with statistical $\pm 1 \sigma$ error bars are shown. $\mathrm{PA}=90^{\circ} \pm 45^{\circ}$ (grey-shaded region) corresponds to the galactic rotation axes tend to be oriented perpendicular with respect to the equatorial plane.

size was chosen to be $20^{\circ}$ ( 9 bins) in all these tests. The statistically poor bins (number of solution $<5$ ) are omitted in the analysis. The conditions for anisotropy are the following: the chi-square probability $\mathrm{P}\left(\chi^{2}\right)<0.050$, correlation coefficient $\mathrm{C} / \sigma(\mathrm{C})>1$, first order Fourier coefficient $\Delta_{11} / \sigma\left(\Delta_{11}\right)>1$ and the first order Fourier probability $\mathrm{P}\left(>\Delta_{1}\right)<0.150$ as used by Godlowski (1993, 1994). Table 2 lists the statistical parameters for the total samples and subsamples.

In the histograms (see Figs. 4-7), a hump at $90^{\circ} \pm 45^{\circ}$ (greyshaded region) suggests that the galactic planes of galaxies tend to lie in the equatorial plane. In other words, the rotation axes of galaxies tend to be oriented perpendicular with respect to the equatorial plane when there is excess number of solutions in the grey-shaded region in the histograms.

All three statistical tests show isotropy in the T modes of the total galaxies. Thus, no preferred alignment is noticed for the $\mathrm{T}$ mode (solid circles in Fig. 4a). Interestingly, all three statistical tests show anisotropy in the total L mode. The chisquare and Fourier probabilities $\left(\mathrm{P}\left(>\chi^{2}\right), \mathrm{P}\left(>\Delta_{1}\right)\right)$ are found $1.5 \%$ (<5\% limit) and 8.5\% (<15\% limit), respectively (Table $2)$. The auto correlation coefficient $(\mathrm{C} / \mathrm{C}(\sigma))$ turned $-3.2(>>1)$. The $\Delta_{11} / \sigma\left(\Delta_{11}\right)$ value is found to be negative at $\sim 2 \sigma$ level, suggesting that the rotation axes of $\mathrm{L}$ mode galaxies tend to be oriented the equatorial plane. Three humps at $50^{\circ}(>1.5 \sigma)$, $90^{\circ}(>2 \sigma)$ and $130^{\circ}(1.5 \sigma)$ can be seen (Fig. 4a). All these humps lie in the grey-shaded region. We checked the binning 
biasness in the statistics by increasing the number of bins to 12 and 16. A similar statistical result is found for both modes. Fig. 4b shows the PA-distribution histogram for the total sample in 18 bins. The L mode shows three significant humps \{ in the grey-shaded region, supporting the above mentioned result. Thus, we conclude isotropy for trailing whereas anisotropy for leading modes in the total sample.

In the spirals, the chi-square and auto correlation tests show isotropy for both L \& T modes. The first order Fourier probability is found greater than $35 \%$, suggesting no preferred alignment. However, the $\Delta_{11}$ value exceeds $1 \sigma$ limit $(-1.2 \sigma)$ in the trailing spirals. A hump at $90^{\circ}$ is not enough to turn the $\Delta_{11} / \sigma\left(\Delta_{11}\right)>1.5$ (Fig. 5a). Similarly, a hump at $150^{\circ}$ is not enough to make the $\Delta_{11} / \sigma\left(\Delta_{11}\right)>1.5$ in the leading spirals. Hence, the preferred alignment is not profounded in both the leading and trailing spirals. Thus, we conclude a random orientation in the PA-distribution.
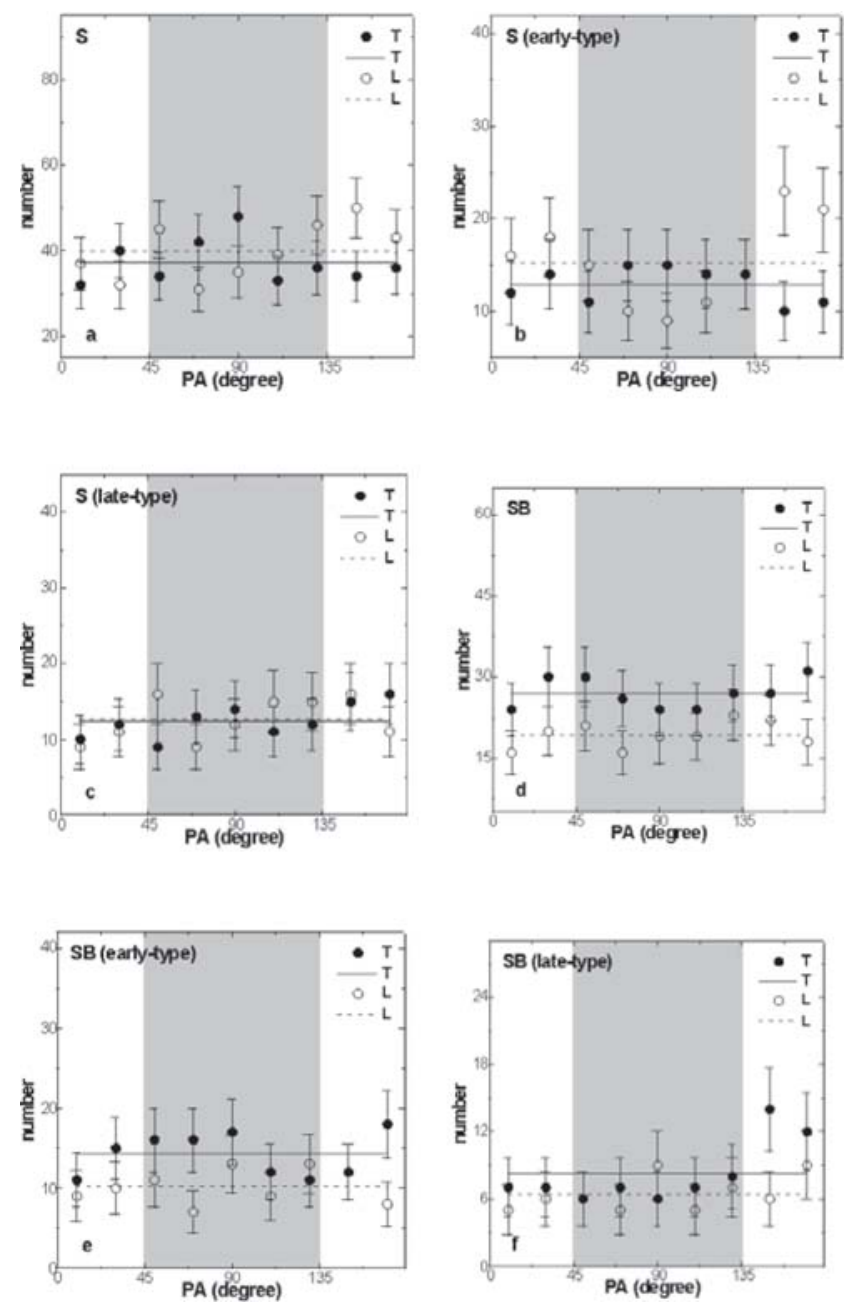

Figure 5: The equatorial PA-distribution of trailing and leading arm galaxies in the spirals (a), early-type spirals (b), late-type spirals (c), barred spirals (d), early-type barred spirals (e) and late-type barred spirals (f). The symbols, error bars, dashed lines and the explanations are analogous to Fig. 4.

Early- and late-type $\mathrm{T}$ spirals show isotropy in all three statistical tests (Table 2). No humps and the dips are seen in the histograms (solid circles in Fig. 5b,c). Thus, T spirals show a random alignment in the PA-distribution. In the subsample SE, all three statistical tests show anisotropy (Table 2). Two significant humps at $>150^{\circ}$ cause the first order Fourier coefficient $\left(\Delta_{11}\right)>+2.5 \sigma$ (hollow circle in Fig. $5 b)$. Thus, a preferred alignment is noticed in the early-type $L$ spirals: the galactic rotation axes tend to lie in the equatorial plane. The late-type leading spirals show a random alignment.

The spiral barred galaxies show a random alignment in both $\mathrm{L}$ \& $\mathrm{T}$ modes. In Fig. 5d, no deviation from the expected distribution can be seen. All three statistical tests support this result (Table 2). A similar result is found for the earlytype SB galaxies in both modes (Table 2, Fig. 5e).

Thus, the late-type T \& L mode SB galaxies show preferred and random alignments, respectively.

\section{Radial velocity}

The subsamples RV1 and RV2 show isotropy in all three statistical tests (Table 2). No humps or dips can be seen in Figs. 6a,b. Thus, the galaxies having radial velocity in the range $3,000 \mathrm{~km} / \mathrm{s}$ to $4,000 \mathrm{~km} / \mathrm{s}$ show a random alignment for both L \& T modes modes.

The humps at $90^{\circ}(>2 \sigma)$ and $110 \mathrm{o}(>2 \sigma)$ are found in $\mathrm{L}$ and $\mathrm{T}$ RV3 galaxies, respectively (Fig. 6c). These two significant humps lead the subsample show anisotropy in the statistical tests (Table 2). The $\Delta_{11}$ values are found negative at $\geq 1.5$ level, suggesting a similar preferred alignment for both modes: the galaxy rotation axes tend to be directed perpendicular to the equatorial plane.
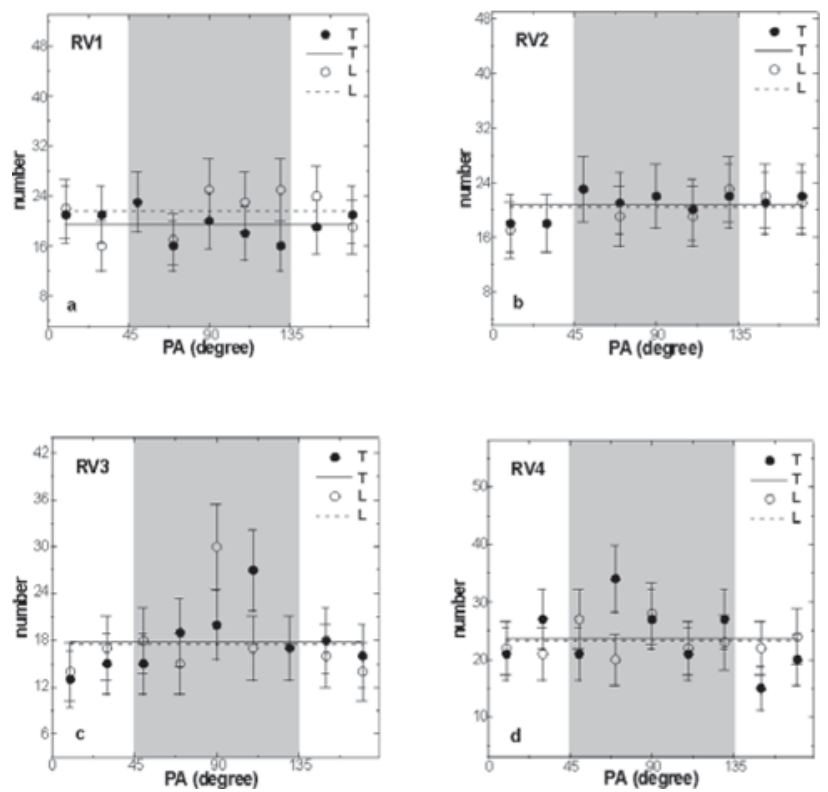

Figure 6: The equatorial PA-distribution of $\mathrm{L} \& \mathrm{~T}$ mode galaxies in RV1 (a), RV2 (b), RV3 (c) and RV4 (d). The abbreviations are listed in Table 1 . The symbols, error bars, dashed lines and the explanations are analogous to Fig. 4.

A hump at $70^{\circ}(>1.5 \sigma)$ and a dip at $150^{\circ}(\sim 2 \sigma)$ cause T mode RV4 galaxies to show anisotropy in all three statistical tests (Fig. 6d). Thus, T subsample having radial velocity in the range $4,500 \mathrm{~km} / \mathrm{s}$ to $5,000 \mathrm{~km} / \mathrm{s}$ show a similar alignment as 
Table 2: Statistics of the PA-distribution of galaxies in the total sample and subsamples (first column). The second, third, fourth and fifth columns give the chi-square probability $\left(\mathrm{P}\left(>\chi^{2}\right)\right)$, correlation coefficient $(\mathrm{C} / \mathrm{C}(\sigma))$, first order Fourier coefficient $\left(\Delta_{11} / \sigma\left(\Delta_{11}\right)\right.$ and first order Fourier probability $\mathrm{P}\left(>\Delta_{1}\right)$, respectively. The last four columns repeat the previous columns.

\begin{tabular}{lcccccccc}
\hline sample & \multicolumn{3}{c}{ Trailing } & \multicolumn{5}{c}{ Leading } \\
& $\mathrm{P}\left(>\chi^{2}\right) \mathrm{C} / \mathrm{C}(\sigma)$ & $\Delta_{11} / \sigma\left(\Delta_{11}\right) \mathrm{P}\left(>\Delta_{1}\right) \mathrm{P}\left(>\chi^{2}\right)$ & $\mathrm{C} / \mathrm{C}(\sigma)$ & $\Delta_{11} / \sigma\left(\Delta_{11}\right) \mathrm{P}\left(>\Delta_{1}\right)$ \\
\hline total & 0.666 & +0.0 & -0.9 & 0.381 & 0.015 & -3.2 & -1.9 & 0.085 \\
S & 0.511 & -0.7 & -1.2 & 0.434 & 0.225 & +0.4 & +0.8 & 0.383 \\
SE & 0.973 & +0.1 & -0.9 & 0.569 & 0.031 & +2.0 & +2.8 & 0.015 \\
SL & 0.234 & +0.5 & +0.8 & 0.209 & 0.460 & -0.1 & -0.5 & 0.345 \\
SB & 0.729 & +0.3 & +1.0 & 0.454 & 0.285 & -1.0 & -0.2 & 0.497 \\
SBE & 0.739 & +0.1 & -0.5 & 0.566 & 0.230 & -0.7 & +0.1 & 0.521 \\
SBL & 0.043 & +1.8 & +1.7 & 0.046 & 0.620 & -0.9 & -0.2 & 0.872 \\
RV1 & 0.910 & +0.3 & +0.8 & 0.362 & 0.369 & -0.9 & -0.6 & 0.285 \\
RV2 & 0.790 & +0.3 & -1.0 & 0.496 & 0.925 & -0.4 & -0.2 & 0.887 \\
RV3 & 0.050 & +1.6 & -1.5 & 0.083 & 0.033 & -1.8 & -2.3 & 0.046 \\
RV4 & 0.043 & -2.3 & -1.5 & 0.116 & 0.636 & +0.2 & -0.7 & 0.692 \\
Gr2 & 0.455 & +0.6 & +0.8 & 0.861 & 0.033 & -1.8 & +1.7 & 0.116 \\
Gr5 & 0.033 & -1.4 & -2.0 & 0.085 & 0.516 & +0.4 & -0.4 & 0.548 \\
\hline
\end{tabular}

shown by the subsample RV3: galactic planes of galaxies tend to lie in the equatorial plane. The L mode RV4 subsample shows a random alignment (Table 2, Fig. 6d).

\section{Groups}

We do not study PA-distribution of L \& T subsamples in the groups Gr1, Gr3, Gr4 and Gr6 because of poor statistics (number $<50$ ). Fortunately, a very good correlation between the $\mathrm{L} \& \mathrm{~T}$ subsamples is noticed in these groups. The chirality is not violated in these groups. We study the PA-distribution of $L \&$ T subsamples in the groups Gr2 and Gr5, where the chiral symmetry seems to be violated. In addition, the statistics is relatively better in these two groups.
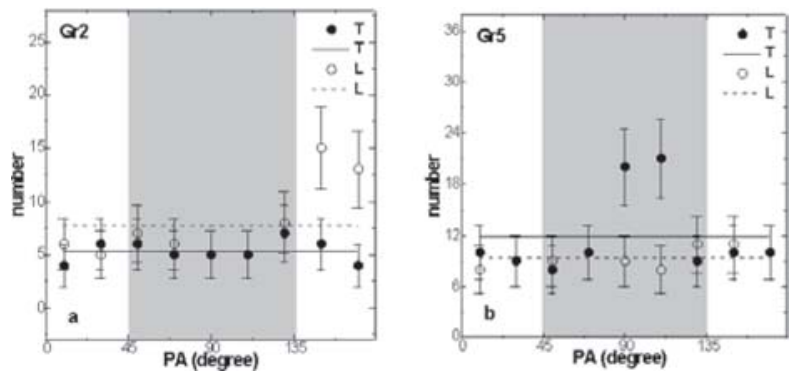

Figure 7: The equatorial PA-distribution of L \& T mode galaxies in the groups Gr2 and Gr5. The abbreviations are listed in Table 1. The symbols, error bars, dashed lines and the explanations are analogous to Fig. 4.

In the group Gr2, L mode dominates. In this group, L mode shows a preferred alignment whereas T mode shows a random alignment in the PA-distribution. All three statistical tests suggest anisotropy in the leading arm galaxies (Table 2). The humps at $>150^{\circ}$ cause the $\Delta_{11}$ value to be positive at $>1.5 \sigma$ level (Fig. 7a), suggesting that the rotation axes of $\mathrm{L}$ mode galaxies in Gr2 tend to be oriented parallel the equatorial plane. The T mode dominates in the group Gr5. Interestingly, a preferred alignment is noticed in the PA-distribution. In Fig. 7b, two significant humps at $900(\sim 2 \sigma)$ and $110^{\circ}(>2 \sigma)$ can be seen. These humps lead the subsample (trailing Gr5) to show anisotropy in the statistical tests (Table 2). No preferred alignment is noticed in $\mathrm{L}$ mode galaxies in this group.

\section{DISCUSSION}

Fig. 8a shows a comparison between the number and position angle $\left(\Delta_{11} / \sigma\left(\Delta_{11}\right)\right)$ distribution of leading and trailing mode galaxies in the total sample and subsamples. This plot deals the possible correlation between the chirality (non-chirality) and the random (preferred) alignment in the subsamples. The grey-shaded region represents the region of isotropy and chirality for the $\Delta_{11} / \sigma\left(\Delta_{11}\right)$ and $\Delta(\%)$, respectively.

Twenty five (out of 39,64\%) subsamples lie in the greyshaded region (Fig. 8a), suggesting a good agreement between the chiral property and the random alignment of the rotation axes of galaxies. In four subsamples (SE, SBL, Gr2 and Gr5), a good correlation between the preferred alignment and the achiral (i.e., non-chiral) property is noticed (Fig. 8a). Thus, It is found that the random alignment of the PAs of galaxies lead the existence of chiral property of galaxies.
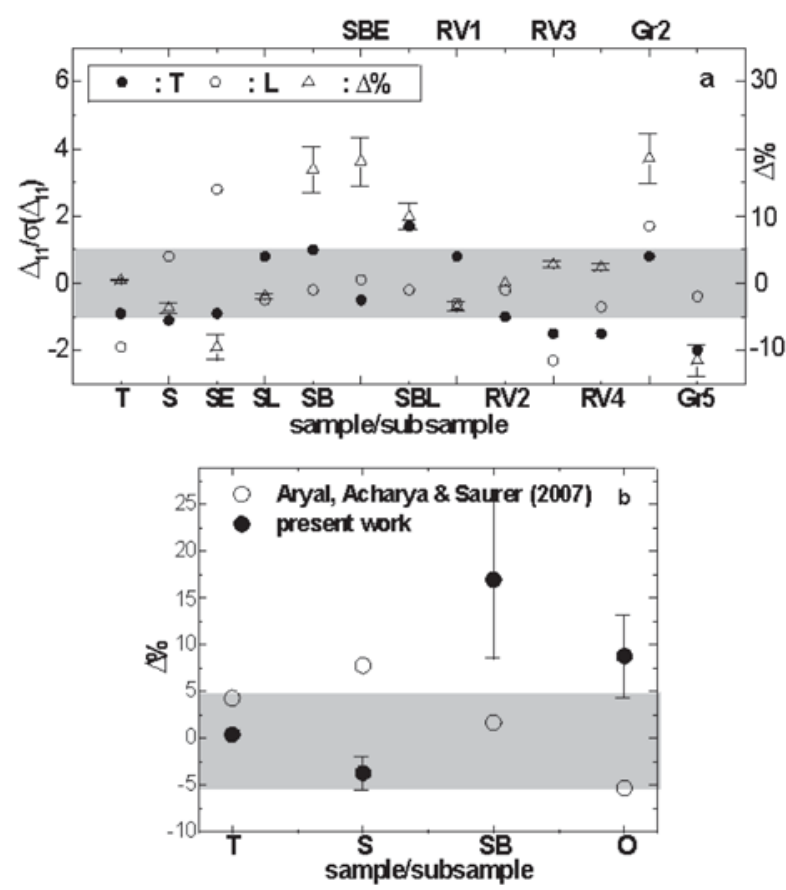

Figure 8: (a) A comparison between the number ( $\Delta \%)$ and the position angle $\left(\Delta_{11} / \sigma\left(\Delta_{11}\right)\right)$ distribution of leading and trailing mode galaxies in the total sample and subsamples. (b) A comparison with the previous work (Aryal, Acharya \& Saurer 2007). Error bars as in Fig. 2.

Here we discuss our results with the results obtained by Aryal et al. (2007). Aryal et al. (2007, hereafter Paper 1) compiled a database of 667 leading and trailing mode galaxies in the LSC $(\mathrm{RV}<3,000 \mathrm{~km} / \mathrm{s})$ and studied the chiral property in the total sample and 17 subsamples. In the present study, the database is compiled from the field galaxies having RV in the range 3,000 to 5,000 km/s. Thus, we are moving deep inside the sky and studying the existence of chiral property in the present work. The distribution of the $\mathrm{L} \& \mathrm{~T}$ mode galaxies in the LSC (Paper 1) as well as in the field (present work) is found homogeneous (Fig. 8b). The difference between the $\mathrm{L} \& \mathrm{~T}$ modes is turned well within 5\% error limit. This result indicates the fact that the chiral property is a global phenomenon rather than a local phenomenon. For the 
spirals, we noticed no deviation from the chirality. However, Paper 1 found a slight deviation because of the presence of the Virgo cluster. Interestingly, we noticed the violation of chirality in the barred spirals (SB) whereas Paper 1 concluded the SB galaxies as a chiral object. For other morphological types (irregulars, morphologically unidentified galaxies), our result is similar to that of the previous result.

Paper 1 concludes a similar preferred alignment in L \& T modes in the Virgo cluster galaxies: the galactic rotation axes tend to lie in the equatorial plane. We noticed a similar result for the early-type spirals (leading mode), late-type barred spirals (T mode) and the group Gr2 (L mode). An important similarity is found in the past and present study: late-type galaxies show chiral property, whereas this property is found to be violated in the early-type galaxies.

In this way, we noticed few inconsistencies in the present and the previous work. It should be remembered that these inconsistencies are profounded either due to the poor statistics or because of the bias in the sample classification. We combined the database of the Paper 1 and the present work, and studied the statistics. A strong chirality $(\Delta<1 \%$, $\Delta($ a sde $)<0.010$ and $\Delta(a) \%<5 \%)$ is found in the total and the spiral galaxies. However, the $\Delta$ value is found to be greater than $10 \%$ for the barred spirals. Thus, the total and the spiral galaxies having RVs less than $5 \backslash, 000 \mathrm{~km} / \mathrm{s}$ strongly exhibit chiral symmetry. It seems that the chirality loss sequence (spiral '!barred spiral '! elliptical) as predicted by Capozziello and Lattanzi (2006) might be true.

Aryal \& Saurer (2004, 2005b, 2006) and Aryal, Paudel \& Saurer (2007) studied the spatial orientation of galaxies in 32 Abell clusters of BM type I (2004), BM type III (2005b), BM type IIIII (2006) and BM type II (2007) and found a significant preferred alignment in the late-type cluster (BM type II-III, BM type III). They concluded that the randomness decreases systematically in galaxy alignments from early-type (BM type I, II) to late-type (BM type II-III, III) clusters. Thus, the existance of chirality in BM type I cluster, as predicted by Capozziello and Lattanzi (2006) might be true. We noticed a very good correlation between the randomness and the chirality. Probably, this result reveals the fact that the progressive loss of chirality might have some connection with the rotationally supported (spirals, barred spirals) to the randomized (lenticulars, ellipticals) system. Thus, we suspect that the dynamical processes in the cluster evolution (such as late-type clusters) give rise to a dynamical loss of chirality.

\section{CONCLUSION}

We studied the chiral property of 1,621 field galaxies having radial velocity (RV) in the range $3,000 \mathrm{~km} / \mathrm{s}$ to $5,000 \mathrm{~km} / \mathrm{s}$. We assumed random direction for the rotation of galaxies and classified leading and trailing modes using observed patterns (S- and Z-shaped) of the arms in the spiral and barred spirals. The distribution of leading and trailing modes is studied in the total sample and 34 subsamples. To examine non-random effects, the equatorial position angle (PA) distribution of galaxies in the total sample and subsamples are studied. Our results are as follows:

(1) The homogeneous distribution of the trailing and the leading mode galaxies is found, suggesting the existence of chiral symmetry in the field galaxies having RVs 3,000 $\mathrm{km} / \mathrm{s}$ to $5,000 \mathrm{~km} / \mathrm{s}$. The PA-distribution of trailing mode galaxies is found to be random, whereas preferred alignment is noticed for leading galaxies. It is found that the galactic rotation axes of leading mode tend to be oriented perpendicular the equatorial plane.

(2) Leading modes are found more $(3.7 \% \pm 1.8 \%)$ than that of the trailing modes in the spirals whereas a significant dominance $(17 \% \pm 8.5 \%)$ of trailing modes are noticed in the barred spirals. This difference is found $>8 \%$ for the irregulars and the morphologically unidentified galaxies. A random alignment is noticed in the PAdistribution of leading and trailing spirals. In other words, a good correlation between the preferred alignment and the chiral symmetry breaking is found. This result verifies the previous result (Paper 1).

(3) The chirality is found stronger for the late-type spirals (Sc, Scd, Sd and Sm) than that of early-type (Sa, Sab, Sb and $\mathrm{Sbc}$ ). Similar result is found for the late-type barred spirals. Thus, the late-type galaxies are the best candidates for the chiral objects than that of the earlytypes.

(4) A very good correlation between the leading and trailing mode galaxies are found in the RV subsamples. Thus, we conclude that the chirality of field galaxies remain invariant with the global expansion.

(5) The galaxies having RVs $3,000 \mathrm{~km} / \mathrm{s}$ to $4,000 \mathrm{~km} / \mathrm{s}$ show a random alignment for both the leading and the trailing modes. The rotation axes of leading and trailing mode galaxies having 4,000 $<\mathrm{RV}(\mathrm{km} / \mathrm{s}) \leq 4,500$ tend to be oriented perpendicular the equatorial plane.

Aryal et al. (2007) studied the chiral property of the Local Supercluster galaxies $(\mathrm{RV}<3,000 \mathrm{~km} / \mathrm{s})^{14}$. If we include their database, we found a strong $(\Delta<1 \%)$ chiral behavior by the total and the spiral galaxies. However, the $\Delta$ value is found to be greater than $10 \%$ for the barred spirals. Hence, the total and the spiral galaxies having RV $<5,000 \mathrm{~km} / \mathrm{s}$ show chiral property. Thus, the chirality-loss sequence as proposed by Capozziello and Lattanzi (2006) might be true ${ }^{13}$.

\section{ACKNOWLEDGEMENTS}

I thank Innsbruck University, Innsbruck, Austria and Koeln University, Koeln, Germany for arranging collaborative working visit for the period of 4 months during Sept-Dec 2009. In addition I acknowledge Profs. J. Stutzki, W. Saurer, R. Weinberger and V. Ossenkopf for their insightful discussion and suggestion. This research has made use of the NASA/ IPAC Extragalactic Database (NED) which is operated by the Jet Propulsion Laboratory, California Institute of Technology, under contract with the National Aeronautics and Space Administration. 


\section{REFERENCE}

1. Garcia-Garcia, A.M., Cuevas, E. 2006. Phy. Rev. B. 74: 1131.

2. Chang, L. et al. 2007. Phy. Rev. C. 75: 1520.

3. Bagchi, M. et al. 2006. Journal of Astronomy \& Astrophysics. 450: 431.

4. Liddle, A.R., Lyth, D.H. 2000. Cosmological Inflation and LargeScale Structure. Cambridge Univ. Press, Cambridge.

5. Peacock, J.A. 1999. Cosmological Physics, Cambridge Univ. Press, Cambridge.

6. Fall, S.M. 1982. Progress in Cosmology. Proc. of the Oxford Intl. Symp., Oxford, Dordrecht, D. Reidel Publishing Co., 347356.

7. Pasha, I.I. 1985. Sov. Astron. Lett. 11: 1

8. Ostriker, J.P., Peebles, P.J.E., \& Yahil, A. 1974. Astrophysical Journal. 193: L1.

9. Thomasson, M. et al. 1989. Journal of Astronomy \& Astrophysics. 211: 25.

10. Athanassoula, S. 1987. Galactic Dynamics, Princeton Univ. press, Princeton, New Jersey.

11. Sugai, H. \& Iye, M. 1995. Monthly Notices Royal Astron. Soc. 276: 327.

12. Aryal, B., Saurer, W. 2005a. Journal Astronomy \& Astrophysics. 432: 841.

13. Capozziello, S., Lattanzi, A. 2006. Journal Astrophysics \& Space Science. 301: 1-4, 189.

14. Aryal, B., Acharya, S., Saurer, W. 2007. Journal Astrophysics \& Space Science. 307: 369.

15. Abell, G.O., Corwin, H.G., Olowin, R.P. 1989. Astrophysical Journal. Supp. 70: 1

16. Struble, M.F., Rodd, H.J. 1999. Astrophysical Journal. Supp. 125: 355.
17. Brunzendorf, J., Meusinger H. 1999. Journal Astronomy \& Astrophysics. Supp. Ser. 139: 141.

18. Kodaira, K., Okamura, S., Ichikawa, S. 1990. Photometric Atlas of Northern Bright Galaxies, Univ. of Tokyo Press, Tokyo.

19. Nilson, P. 1973. Uppsala General Catalogue of Galaxies, Nova Acta Uppsala University, V:A, Vol.1.

20. Shectman, S.A., Landy, S.D., Oemler, A. et al. 1996. Astrophysical Journal. 470: 172.

21. Nilson, P. 1974. Uppsala Astronomical Observatory Report, 5.

22. Lauberts, A. 1982. ESO/Uppsala Survey of the ESO B Atlas, Garching bei Muenchen.

23. Corwin, H.G., de Vaucouleurs, A., de Vaucouleurs, G. 1985. Univ. Texas Monogr. Astron. 4, 1.

24. de Vaucouleurs, G., de Vaucouleurs, A., Corwin, et al. 1991. Third Reference Catalogue of Bright Galaxies, Springer-Verlag, New York.

25. Aryal, B., Saurer, W. 2004. Journal Astronomy \& Astrophysics. 425: 871.

26. Aryal, B., Saurer, W. 2005b. Journal Astronomy \& Astrophysics. 432: 431.

27. Aryal, B., Saurer, W. 2006. Monthly Notices Royal Astron. Soc. 366: 438.

28. Aryal, B., Paudel, S., Saurer, W. 2007. Monthly Notices Royal Astron. Soc. 379: 1011.

29. Oort, J.H. 1970a. Science. 170: 1363.

30. Peebles P.J.E., 1969. Astrophysical Journal. 155: 393.

31. Aryal, B., Saurer, W. 2000. Journal Astronomy Astrophysics Lett. 364: L97.

32. Godlowski, W. 1993. Monthly Notices Royal Astron. Soc.265: 874. 